\title{
Plain endotracheal tube insertion carries greater risk for malpositioning than does reinforced endotracheal tube insertion in females
}

\author{
Jin-Hee Han ${ }^{1}$, Seung-Hoon Lee ${ }^{1}$, Young-Jin Kang ${ }^{1}$, and Jong-Man Kang ${ }^{2}$ \\ Department of Anesthesiology and Pain Medicine, ${ }^{1}$ Kyung Hee University Hospital, ${ }^{2}$ Kyung Hee University Hospital at Gangdong, \\ Seoul, Korea
}

Malpositioning of an endotracheal tube within the airway can lead to serious complications, such as endobronchial intubation, which may cause collapse of the nonventilated lung and barotrauma of the ventilated lung or vocal cord paralysis and accidental extubation [1].

We retrospectively identified 204 (54 plain tubes and 54 reinforced tubes in males; 36 plain and 60 reinforced tubes in females) chest X-rays of patients with endotracheal tubes suitable for analysis by examining the radiology database and selecting the first available postoperative AP chest X-ray with an endotracheal tube in situ. The position of the endotracheal tube tip relative to the carina was measured from postoperative chest X-rays using the Picture Archiving and Communication System (Infinitt
Healthcare Co., Ltd., Seoul, Korea). We also reviewed medical records to obtain the demographic details of the patients and to confirm the use of routine clinical tube placement methods. We defined the appropriate depth of the endotracheal tube from the carina to be $>2 \mathrm{~cm}$ and $\leq 6 \mathrm{~cm}$ [2]. A t-test was performed for statistical analysis within the same gender. A P value of $<0.05$ was considered to be statistically significant.

Demographics and relationships of distal endotracheal tube position in the airway are shown in Table 1. Average age, height, and weight were different between the two groups of males. The distances between the tip of the endotracheal tube to the carina were significantly different between the two groups of female patients.

Table 1. Demographics and Relationship of Distal Endotracheal Tube Position in the Airway

\begin{tabular}{lccccc}
\hline & \multicolumn{2}{c}{ Males } & & Females \\
\cline { 2 - 3 } \cline { 5 - 6 } & Plain tube $(\mathrm{n}=54)$ & Reinforced tube $(\mathrm{n}=54)$ & & Plain tube $(\mathrm{n}=36)$ & Reinforced tube $(\mathrm{n}=60)$ \\
\hline Age $(\mathrm{yrs})$ & $59(28-85)$ & $54(19-82)$ & $62(24-83)$ & $61(34-84)$ \\
Height $(\mathrm{cm})$ & $167.2(5.4)$ & $168.3(6.5)$ & $154.0(8.0)$ & $154.0(5.6)$ \\
Weight $(\mathrm{kg})$ & $60.7(11.4)^{*}$ & $66.7(10.5)$ & $56.3(12.4)$ & $56.3(15.8)$ \\
Fixation at the upper incisor $(\mathrm{cm})$ & $22.8(0.9)$ & $22.8(0.8)$ & $21.6(0.7)$ & $21.5(0.9)$ \\
Tip-carina $(\mathrm{cm})$ & $4.8(1.4)$ & $5.2(1.5)$ & 0 & $2.9(1.6)^{*}$ & $4.5(1.4)$ \\
Endobronchial intubation $(\mathrm{n})$ & 0 & 2 & 5 & 0 \\
Too low $(\leq 2 \mathrm{~cm})$ & 2 & $47(93 \%)$ & $30(80 \%)$ & 1 \\
Acceptable $(>2 \mathrm{~cm}$ and $\leq 6 \mathrm{~cm})$ & 10 & 5 & 0 & $56(87 \%)$ \\
Too high $(>6 \mathrm{~cm})$ & & & & 3
\end{tabular}

*Significantly different $(\mathrm{P}<0.05)$ compared with the reinforced tube group within the same gender. Data are mean (range or SD or percentage) or $\mathrm{n}$.

Corresponding author: Jong-Man Kang, M.D., Department of Anesthesiology and Pain Medicine, Kyung Hee University Hospital at Gangdong, 149, Sangil-dong, Gangdong-gu, Seoul 134-090, Korea. Tel: 82-2-440-6193, Fax: 82-2-440-7808, E-mail: jongmankang@gmail.com

(c) This is an open-access article distributed under the terms of the Creative Commons Attribution Non-Commercial License (http:// creativecommons.org/licenses/by-nc/3.0/), which permits unrestricted non-commercial use, distribution, and reproduction in any medium, provided the original work is properly cited. 
Chest X-rays demonstrated that 29 (14\%) of 204 intubations resulted in inappropriate placement that was not detected by clinical criteria. Endobronchial intubations occurred in five patients; all were females with plain endotracheal tubes.

This study demonstrated that routine oral endotracheal intubation in the operating theater cannot always guarantee optimal tube depth. In addition, we found that insertion of plain endotracheal tubes in female patients carried a greater risk for positioning the tip of the tube near the carina than did the insertion of reinforced tubes in females or the use of either type of tube in males.

Many physicians typically verify the position of the endotracheal tube using simple standard clinical criteria without postintubation confirmation via chest radiograph or fiberoptic bronchoscopic examination. Routine methods to estimate the optimal endotracheal tube length include auscultation of bilateral breath sounds, symmetric chest expansion, and palpation of the endotracheal tube cuff in the suprasternal notch $[1,3,4]$.

Plain tubes are thought to be stiffer than reinforced tubes, and therefore the tips of plain tubes can be placed closer to the carina in females. However, the current study found that this phenomenon was not significant in males. We think that gen- der, height, and age affect the difference in tube depth observed between the two tube groups in an interactive way. Further prospective studies are needed to better define this difference.

This study has several limitations. First, our study was retrospective. It was thought to be beneficial not to order a chest radiograph, which would be needed for a prospective study. Second, most of the patients in this study were elderly and were unextubated postoperatively for ventilator care; therefore, these results might not be easily generalized to all patients, such as those that are young and healthy. Third, the chest X-rays used in this study were not obtained under carefully controlled conditions. Endotracheal tube tip location can markedly change with small alterations in head position, such as flexion or extension of the neck. However, the majority of the chest X-rays included in this study were obtained in the routine supine surgical position because most patients were mechanically ventilated with sedation postoperatively.

In conclusion, the depth of the inserted endotracheal tube should be carefully verified, especially in females who receive plain tubes. Different strategies must be chosen for positioning of endotracheal tubes at an adequate depth according to tube type in females.

\section{References}

1. Owen RL, Cheney FW. Endobronchial intubation: a preventable complication. Anesthesiology 1987; 67: 255-7.

2. Schwartz DE, Lieberman JA, Cohen NH. Women are at greater risk than men for malpositioning of the endotracheal tube after emergent intubation. Crit Care Med 1994; 22: 1127-31.

3. Evron S, Weisenberg M, Harow E, Khazin V, Szmuk P, Gavish D, et al. Proper insertion depth of endotracheal tubes in adults by topographic landmarks measurements. J Clin Anesth 2007; 19: 15-9.

4. Lee BJ, Yi JW, Chung JY, Kim DO, Kang JM. Bedside prediction of airway length in adults and children. Anesthesiology 2009; 111: 556-60. 